Article

\title{
Oxadiazole-Based Highly Efficient Bipolar Fluorescent Emitters for Organic Light-Emitting Diodes
}

\author{
Qiong $\mathrm{Wu}^{1,2}$, Ramanaskanda Braveenth ${ }^{1}{ }^{(\mathbb{D}}$, Heng Qiang Zhang ${ }^{2}$, Il-Ji Bae ${ }^{3}$, Miyoung Kim ${ }^{3, *}$ \\ and Kyu Yun Chai ${ }^{1, *}$ \\ 1 Division of Bio-Nanochemistry, College of Natural Sciences, Wonkwang University Iksan City, \\ Chonbuk 570-749, Korea; wuqiong9308@126.com (Q.W.); braveenth.czbt@gmail.com (R.B.) \\ 2 Department of Chemistry, Hebei Normal University for Nationalities, Chengde 067000, China; \\ zhanghengqiang80@163.com \\ 3 Nano-Convergence Research Center, Korea Electronics Technology Institute, Jeonju 54853, Korea; \\ ijbae@keti.re.kr \\ * Correspondence: miy1kim@keti.re.kr (M.K.); geuyoon@wonkwang.ac.kr (K.Y.C.); \\ Tel.: +82-63-219-0011 (M.K.); +82-63-850-6230 (K.Y.C.); \\ Fax: +82-63-219-0049 (M.K.); +82-63-841-4893 (K.Y.C.)
}

Received: 14 March 2018; Accepted: 5 April 2018; Published: 7 April 2018

\begin{abstract}
In this study, a series of bipolar fluorescence emitters named 2DPAc-OXD, DPAc-OXD, 2PTZ-OXD and PTZ-OXD were designed and synthesized with excellent yields. The characterization of materials was investigated by using nuclear magnetic resonance (NMR) $\left({ }^{1} \mathrm{H},{ }^{13} \mathrm{C}\right)$, mass spectrometry and thermogravimetric analysis (TGA). To investigate device efficiencies, two different OLED devices (Device 1, Device 2) were fabricated with two different host materials (Bepp 2 , DPEPO). The Device 2 with 2PTZ-OXD as fluorescent emitter exhibited excellent power and current efficiencies of $6.88 \mathrm{Lm} / \mathrm{W}$ and $10.10 \mathrm{~cd} / \mathrm{A}$, respectively. The external quantum efficiency of 2PTZ-OXD was around 3.99\% for Device 2. The overall device properties of phenothiazine donor were better than acridine derivatives.
\end{abstract}

Keywords: bipolar; oxadiazole; acridine; phenothiazine; organic light-emitting diodes; fluorescent

\section{Introduction}

Organic light-emitting diodes (OLEDs) have developed rapidly since the first efficient double layer structure device was reported by Tang [1]. They are expected as the promising candidates for next generation flat-panel displays and solid-state lighting sources due to various advantages, such as high-quality color, low energy cost, light weight, and flexibility [2]. Small OLED displays, which are commercially available now, are applied to mobile displays, television and flat panels. Nevertheless, there are still some issues related to device performance and reliability need to improve, especially the efficiency of device with fluorescent emitting materials. To the best of our knowledge, the maximum external quantum efficiency (EQE) of fluorescent material is $8.5 \%$ [3]. The emitting layer functions as the recombination site of holes and electrons which are injected from anode and cathode, respectively [4]. Therefore, the development of high efficient fluorescent-emitting material is a key issue for high performance OLED devices.

The charge-recombination factor is dominated by the balance of holes and electrons in the emissive layer and the current balance is directly proportional to the emission [5]. Therefore, emitting layer materials should meet the requirements of energy level matching for charge carrier injection and acceptance of both holes and electrons. Meanwhile, they should possess the properties of electron 
transporting and hole-transporting character to permit the formation of both stable cation and anion radicals, which known as bipolar in nature [4]. Electron-donating units have the potential to transport holes, while electron-accepting units are capable of transporting electrons. Therefore, the most prevalent strategy of achieving bipolar materials is the incorporation of donor-acceptor (D-A) units into the same molecule via a linker to facilitate the injection and transport of both holes and electrons [6]. Recently, A-D and D-A-D types materials are often explored for OLED fluorescent materials [3,7-16] to improve charge balance and reduce driving voltage; they are also widely designed for thermally activated delayed fluorescence (TADF) emitters and host martials for phosphorescence and TADF dopants [17-19]. The emission from A-D and D-A-D type molecular design can originate from intramolecular charge-transfer (ICT) excited states between the donor and acceptor moieties [20,21]. Therefore, judicious combination of the appropriate donor and accepter could facilitate simultaneous manipulation of the highest occupied orbital (HOMO)/the lowest unoccupied orbital (LUMO) levels and the emission color of the bipolar structure molecule [22].

Triazine, oxadiazole, triazole, cyno-substituted benzene and benzothiadiazole are usually used as acceptor units, while carbazole, arylamine, phenothiazine and their derivatives are commonly adopted as the donor moieties for bipolar structures for OLEDs [15-17,20,23-29]. Herein, we utilize phenothiazine and 9,9-diphenyl-9,10-dihydroacridine as the donor unit, while oxadiazole derivatives 2,5-bis(4-bromophenyl)-1,3,4-oxadiazole and 2-(4-bromophenyl)-5-phenyl-1,3,4-oxadiazole are employed as the acceptors to synthesize four distorted A-D and D-A-D type fluorescent materials, 2,5-bis(4-(10H-phenothiazin-10-yl)-1,3,4-oxadiazole (2PTZ-OXD), 2-(4-(10H-phenothiazin-10-yl) -1,3,4-oxadizaole (PTZ-OXD), 2,5-bis(4-(9,9-diphenyl-9,10-dihydroacridine)phenyl)-1,3,4-oxadizaole (2DPAc-OXD) and 2-(4-(9,9-diphenyl-9,10-dihydroacridine)phenyl)-1,3,4-oxadiazole (DPAc-OXD). We also investigated their photophysical properties, thermal stability and performed quantum calculations to evaluate the molecular orbitals distribution for all molecules.

\section{Results and Discussion}

\subsection{Synthesis}

Scheme 1 displays the synthetic process of designed bipolar fluorescent emitters. Compounds 1, 2, 3 and 4 were known and synthesized by following the methods from previously reported literatures [30-32]. 2DPAc-OXD, DPAc-OXD, 2PTZ-OXD and PTZ-OXD were synthesized by using the well-known Buchwald Hartwig cross-coupling reaction between the oxadiazole ( 2 and 4 ) and phenothiazine or 9,9-diphenyl-9,10-dihydroacridine derivatives by using palladium-based catalyst.

\subsection{Thermal Properties}

The thermal stabilities of four fluorescence emitters were studied on the basis thermal decomposition temperature at $5 \%$ weight reduction and were measured by using thermo gravimetric analysis (TGA). All four emitters, which are depicted in Figure 1 exhibited higher decomposition temperature over $358{ }^{\circ} \mathrm{C}$. The acridine donor-based emitters, DPAc-OXD and 2DPAc-OXD showed thermal stability of 375 and $455^{\circ} \mathrm{C}$, respectively. The above values are higher than that of phenothiazine donor based PTZ-OXD $\left(358^{\circ} \mathrm{C}\right)$ and 2PTZ-OXD $\left(419^{\circ} \mathrm{C}\right)$ due to its higher rigidity when compare to acridine donor-based molecules (Table 1). Additionally, we noticed that two site molecules (2PTZ-OXD, 2DPAc-OXD) were excellent with their thermal strength while the thermal strength of single site molecules (PTZ-OXD, DPAc-OXD) were lower. 

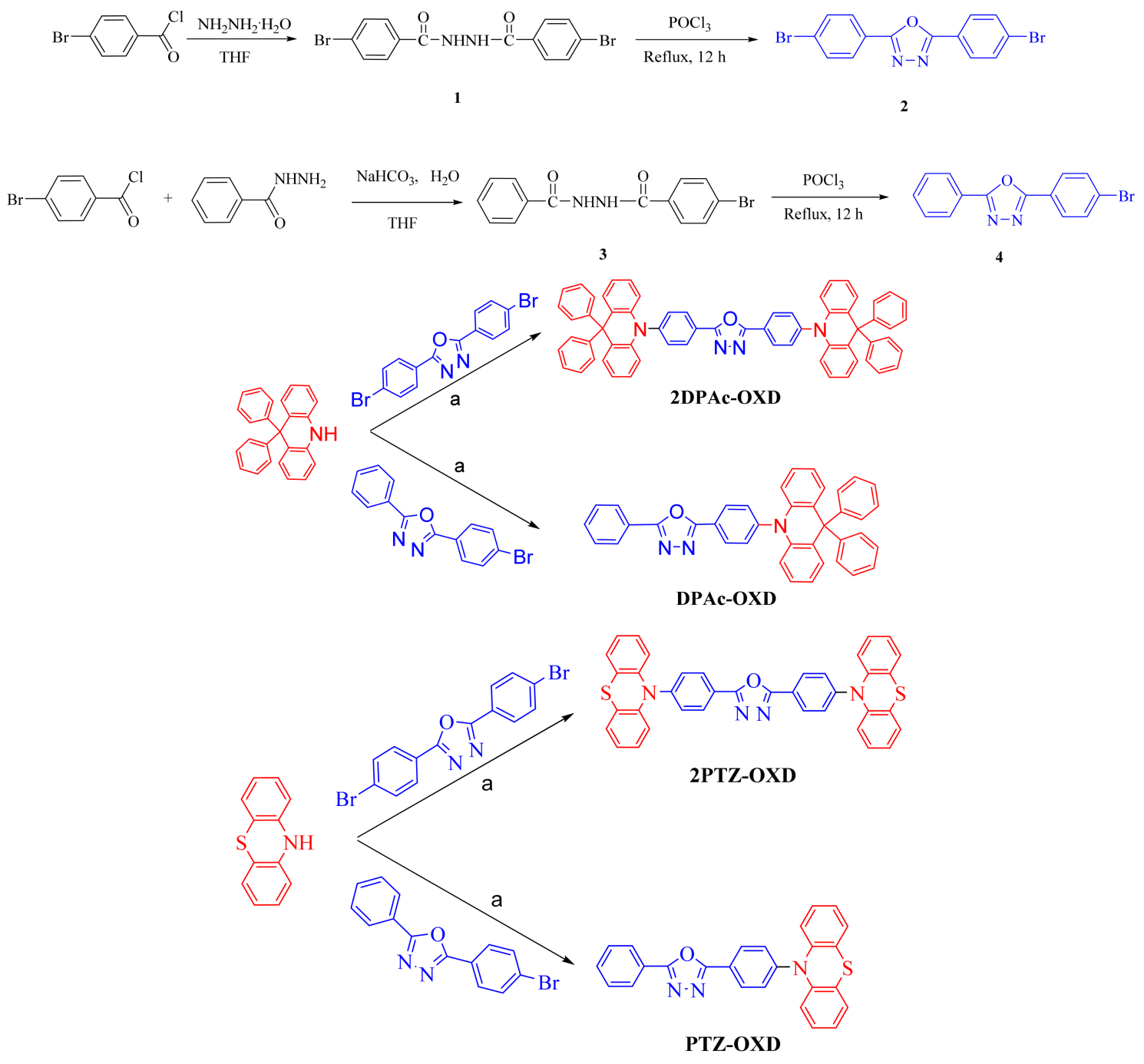

Scheme 1. Synthesis of 2,5-bis(4-(9,9-diphenyl-9,10-dihydroacridine)phenyl)-1,3,4-oxadizaole (2DPAc-OXD), 2-(4-(9,9-diphenyl-9,10-dihydroacridine)phenyl)-1,3,4-oxadiazole (DPAc-OXD), 2,5-bis(4-(10H-phenothiazin-10-yl)-1,3,4-oxadiazole (2PTZ-OXD) and 2-(4-(10H-phenothiazin -10-yl)-1,3,4-oxadizaole (PTZ-OXD). Reagents and conditions (a): $\mathrm{Pd}(\mathrm{OAc})_{2}, \mathrm{~K}_{2} \mathrm{CO}_{3}, t$ - $\mathrm{Bu}_{3} \mathrm{P}$, anhydrous toluene, $110^{\circ} \mathrm{C}$.

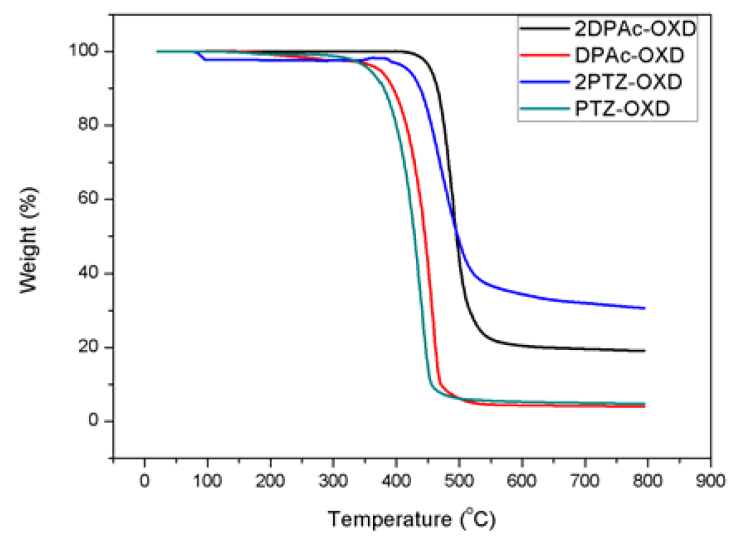

Figure 1. Thermal decomposition temperature (by thermogravimetric analysis, TGA) of fluorescent emitters. 
Table 1. Thermal and physical properties of fluorescent emitters.

\begin{tabular}{|c|c|c|c|c|c|c|c|}
\hline Dopant & $\begin{array}{l}T_{d}{ }^{\mathrm{a}} \\
\left({ }^{\circ} \mathrm{C}\right)\end{array}$ & $\begin{array}{c}\text { UV-vis b } \\
(\mathrm{nm})\end{array}$ & $\underset{(\mathrm{nm})}{P L \max }$ & $\begin{array}{c}\text { HOMO }^{d} \\
\quad(e V)\end{array}$ & $\begin{array}{c}\text { LUMO }^{\mathrm{e}} \\
\quad(\mathrm{eV})\end{array}$ & $\begin{array}{l}E_{g} \mathrm{f} \\
(\mathrm{eV})\end{array}$ & $\begin{array}{l}E_{T} \mathrm{~g} \\
(\mathrm{eV})\end{array}$ \\
\hline PTZ-OXD & 358 & 376 & 500 & -5.48 & -2.18 & 3.30 & 2.48 \\
\hline 2PTZ-OXD & 419 & 384 & 512 & -5.48 & -2.25 & 3.23 & 2.42 \\
\hline DPAc-OXD & 375 & 396 & 435 & -5.69 & -2.56 & 3.13 & 2.85 \\
\hline 2DPAc-OXD & 455 & 402 & 442 & -5.68 & -2.60 & 3.08 & 2.81 \\
\hline
\end{tabular}

a Decomposition temperature at $5 \%$ weight reduction; ${ }^{\mathrm{b}}$ On set UV absorption; ${ }^{\mathrm{c}}$ Photoluminescence emission (PL);

${ }^{d}$ Highest occupied molecular orbital energy (HOMO); ${ }^{\mathrm{e}}$ Lowest unoccupied molecular orbital energy (LUMO);

${ }^{\mathrm{f}}$ Band gap energy; ${ }^{\mathrm{g}}$ Triplet energy.

\subsection{Photophysical and Electrochemical Properties}

The photophysical properties were analysed by using UV-visible and photoluminescence spectral measurements. UV-Vis spectra showed on set values of 376, 384, 396 and $402 \mathrm{~nm}$ for PTZ-OXD, 2PTZ-OXD, DPAc-OXD and 2DPAc-OXD, respectively which were used to calculate the band gap energies of four emitters (Figure 2). The above values are summarized in Table 1 . The triplet energy of PTZ-OXD, 2PTZ-OXD, DPAc-OXD and 2DPAc-OXD were 2.48, 2.42, 2.85 and $2.81 \mathrm{eV}$, respectively. Those above values were evaluated from phosphorescence emission spectrum according to literature [33]. The triplet energy values were matched with their photoluminescence (PL) emission of 500 (PTZ-OXD), 512 (2PTZ-OXD), 435 (DPAc-OXD) and $442 \mathrm{~nm}$ (2DPAc-OXD). When we noticed the PL values of phenothiazine donor-based molecules, PTZ-OXD and 2PTZ-OXD were revealed bathochromic shift related to their lower triplet energy. HOST material is one of the key factors for enhancing the device efficiencies by controlling holes and electrons balance at the emission layer. We have fabricated two different OLED devices with different host materials of Bis[2-(2-pyridinyl)phenolato]beryllium(II) (Bepp 2 ) and Bis[2-(diphenylphosphino)phenyl] ether oxide (DPEPO), which have higher triplet energy of 2.70 and $2.99 \mathrm{eV}$ for latest [34,35]. DPEPO host material has enough potential to prevent the energy transferring from dopant to host due to its higher triplet energy than our fluorescence emitters. In the case of Bepp 2 host material, it can effectively work with PTZ-OXD and 2PTZ-OXD due to their lower triplet energies.
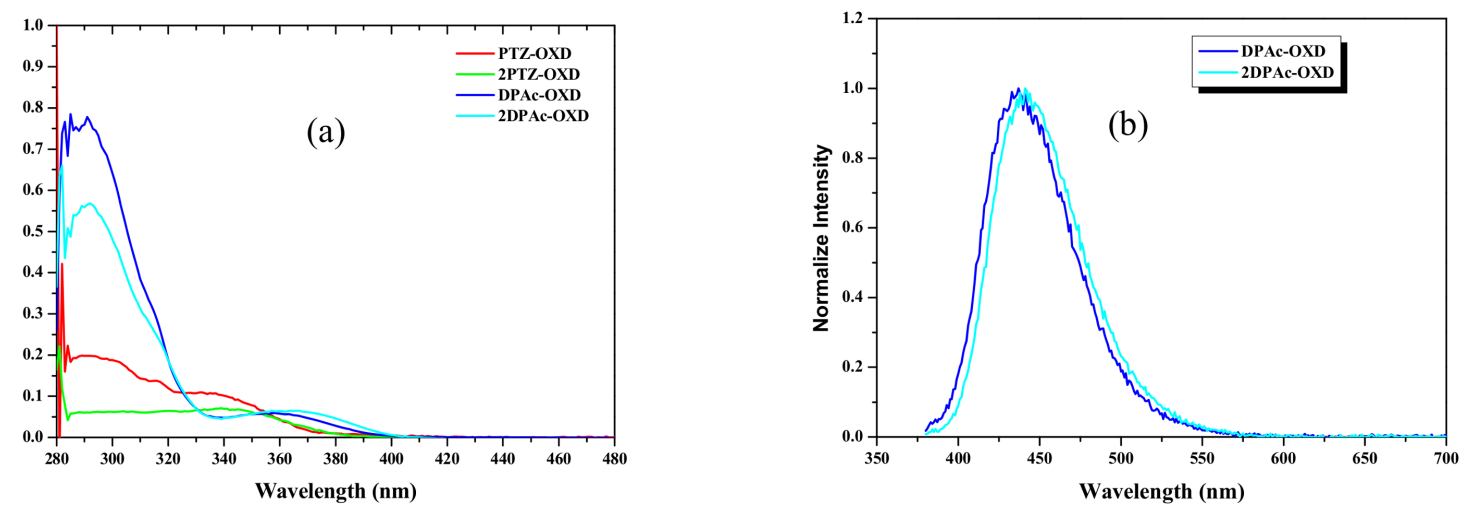

Figure 2. (a) UV-Vis absorption spectra (b) Photoluminescent spectra of fluorescent emitters.

The electrochemical properties were analysed on the basis of HOMO and LUMO energies. The HOMO value of acridine donor based DPAC-OXD and 2DPAC-OXD were the same with $-5.68 \mathrm{eV}$, while phenothiazine donor based PTZ-OXD and 2PTZ-OXD were almost identical to each other $(-5.48 \mathrm{eV})$. Consequently, LUMO energy values were calculated by subtracting the band gap energies from HOMO energies and the values were $-2.18,-2.25,-2.56$ and $-2.60 \mathrm{eV}$ for PTZ-OXD, 2PTZ-OXD, DPAc-OXD and 2DPAc-OXD, respectively. The frontier molecular energies contributed to constructing the device structure substantiated with proper energy flow with adjacent layers. 
Density functional theory (DFT) calculations were investigated by using Gaussian 9 program with TD-SCF method B3LYP and 6-31G basis set [36]. The frontier molecular orbital (FMO) distributions are shown in Figure 3. All four materials had clear HOMO and LUMO separations and we did not notice any overlapping between the donor and acceptor. HOMO of all molecules was distributed over phenothiazine and acridine moieties due to their strong electron donating nature, which could be explained by the electron localization on donor molecules. The electron delocalization was observed at oxadiazole due to its electron withdrawing nature. The clear separation of FMOs are supported by their lower singlet-triplet energy gap difference of $0.35,0.29,0.46$ and $0.44 \mathrm{eV}$ for PTZ-OXD, 2PTZ-OXD, DPAc-OXD and 2DPAc-OXD, respectively (Table 2). The rotation angle between phenothiazine donor and oxadiazole was around $78^{\circ}$, but acridine and oxadiazole showed higher angle of $81^{\circ}$ as bulky phenyl groups attached with $s p^{3}$ carbon atom of acridine molecule. The above results indicate that four A-D and D-A-D type fluorescence emitters were successfully constructed with bipolar charge transfer properties.

Compound

PTZ-OXD

2PTZ-OXD

DPAC-OXD

2DPAc-OXD
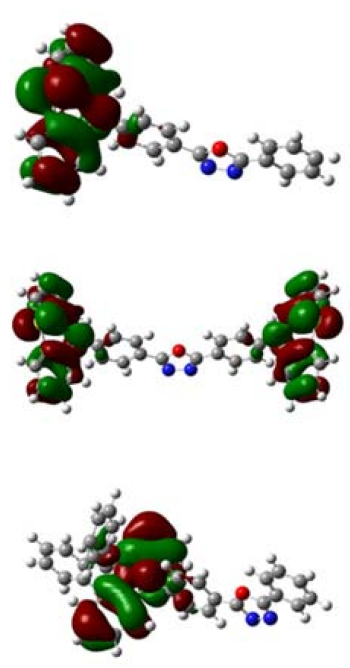

HOMO
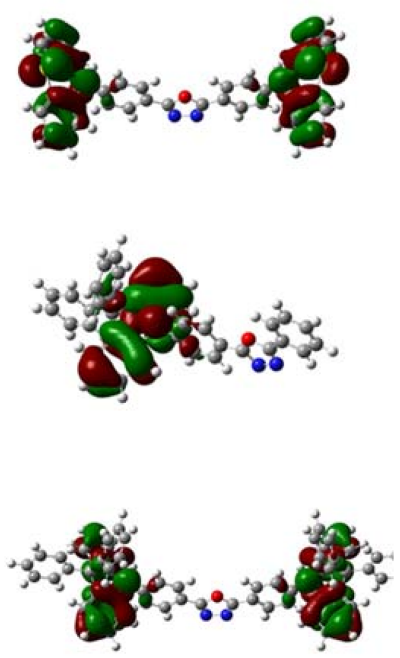

LUMO
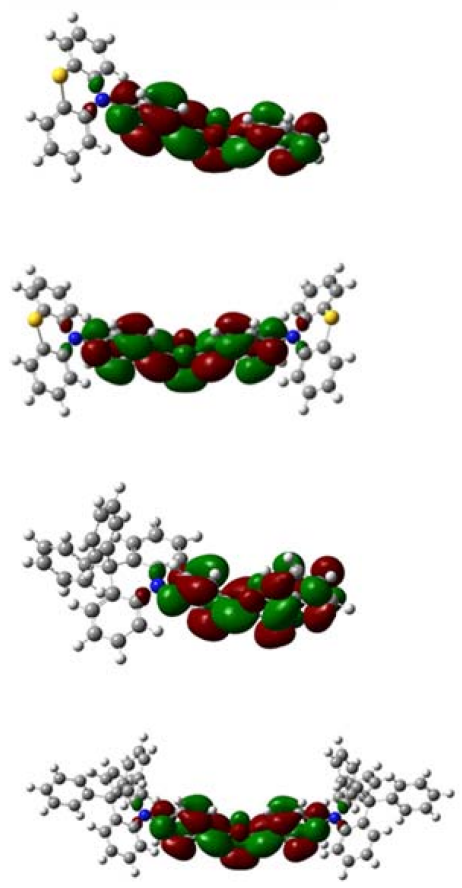

Figure 3. Calculated Frontier molecular orbital distribution.

Table 2. Calculation data of four dopant materials.

\begin{tabular}{ccccc}
\hline Calculation Values & PTZ-OXD & 2PTZ-OXD & DPAc-OXD & 2DPAc-OXD \\
\hline $\mathrm{S}_{1}(\mathrm{eV})^{\mathrm{a}}$ & 3.71 & 3.63 & 3.83 & 3.78 \\
$\mathrm{~T}_{1}(\mathrm{eV})^{\mathrm{b}}$ & 3.36 & 3.34 & 3.37 & 3.34 \\
$\Delta E_{\mathrm{ST}}(\mathrm{eV})^{\mathrm{c}}$ & 0.35 & 0.29 & 0.46 & 0.44 \\
$\mathrm{D}-\mathrm{A}$ rotation $\left({ }^{\circ}\right)$ & 77.05 & 78.91 & 81.26 & 81.70 \\
HOMO $(\mathrm{eV})$ & -5.975 & -5.485 & -5.695 & -5.685 \\
LUMO $(\mathrm{eV})$ & -2.185 & -2.255 & -2.565 & -2.605 \\
\hline
\end{tabular}

${ }^{a}$ Singlet energy; ${ }^{\mathrm{b}}$ Triplet energy; ${ }^{\mathrm{c}}$ Triplet and singlet energy difference. 


\subsection{Device Characteristics}

The device characteristics were studied after fabricating OLED devices with two different host materials (Figure 4). The device structures were (Device 1) ITO (150nm)/ NPB (20 nm)/ TCTA (10 nm)/ $\mathrm{mCP}(10 \mathrm{~nm}) / \mathrm{Bepp}_{2}:$ dopant 6\%(10 nm)/TPBi $(55 \mathrm{~nm}) / \mathrm{Liq}(3 \mathrm{~nm}) / \mathrm{Al}(100 \mathrm{~nm})$ and (Device 2) ITO $(150 \mathrm{~nm}) / \mathrm{NPB}(30 \mathrm{~nm}) / \mathrm{mCP}(10 \mathrm{~nm}) / \mathrm{DPEPO}$ dopant 6\%(15 nm)/DPEPO (10 nm)/ TPBi $(40 \mathrm{~nm}) /$ Liq (3 nm)/ Al (100 nm).
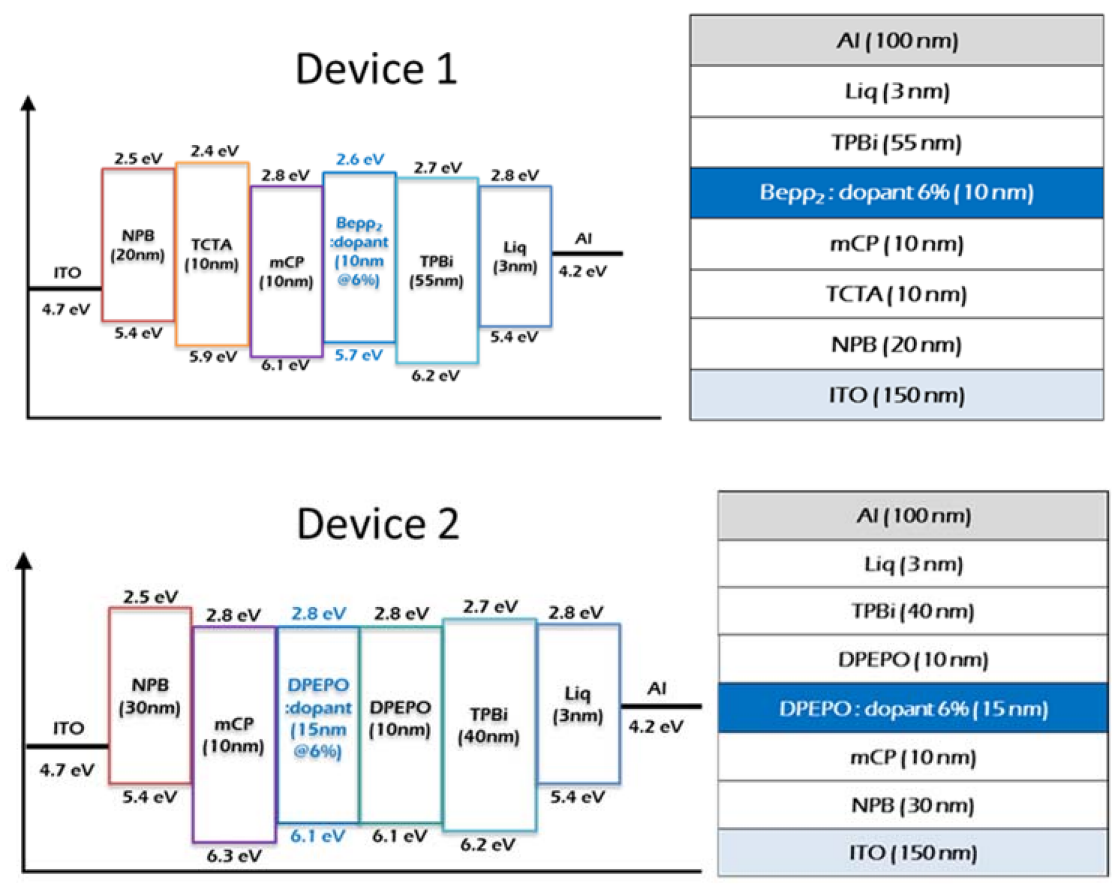

Figure 4. The fabricated device structures of 1 and 2 based on fluorescent emitters.

The turn on voltages of device 1 were between 4.1 to $4.4 \mathrm{~V}$, which was lower than that of device 2 (5.6 to 6.2 V). PTZ-OXD and 2PTZ-OXD showed lower driving voltage than acridine based DPAc-OXD and 2DPAc-OXD, which proved that phenothiazine donor in cooperating with oxadiazole acceptor can enhance the device efficiencies (Figure 5). The current efficiency of device 1 was better than device 2, but 2PTZ-OXD based Device 1 exhibited current efficiency of $9.20 \mathrm{~cd} / \mathrm{A}$ while Device 2 was $10.10 \mathrm{~cd} / \mathrm{A}$. The above value is higher than reported current efficiency of fluorescence-based emitters in OLEDs. 2PTZ-OXD based Device 1 showed higher power efficiency of $6.88 \mathrm{Lm} / \mathrm{W}$, which is related with lower driving voltage of $5.9 \mathrm{~V}$. PTZ-OXD revealed power efficiency of $3.59 \mathrm{Lm} / \mathrm{W}$ for its higher driving voltage $(6.8 \mathrm{~V})$. The current and power efficiencies of acridine based DPAc-OXD and 2DPAc-OXD were lower than phenothiazine-based molecules which is an evidence that acridine and oxadiazole do not provide balance charge transfer system due to strong electron donating ability of acridine molecule than that of phenothiazine. Consequently, external quantum efficiency (EQE) of 2PTZ-OXD based Device 1 was $3.38 \%$ and Device 2 was 3.99\%. The EQE value of PTZ-OXD, DPAc-OXD and 2DPAc-OXD were $1.94 \%, 1.84 \%$ and $1.81 \%$ respectively (Table 3 ). However, the efficiencies at high current density were dropped from peak value due to efficiency roll-off. Which attributed to quenching mechanism such as molecular exciton annihilation occurs at high current densities $[37,38]$. 


\section{DEVICE 1}
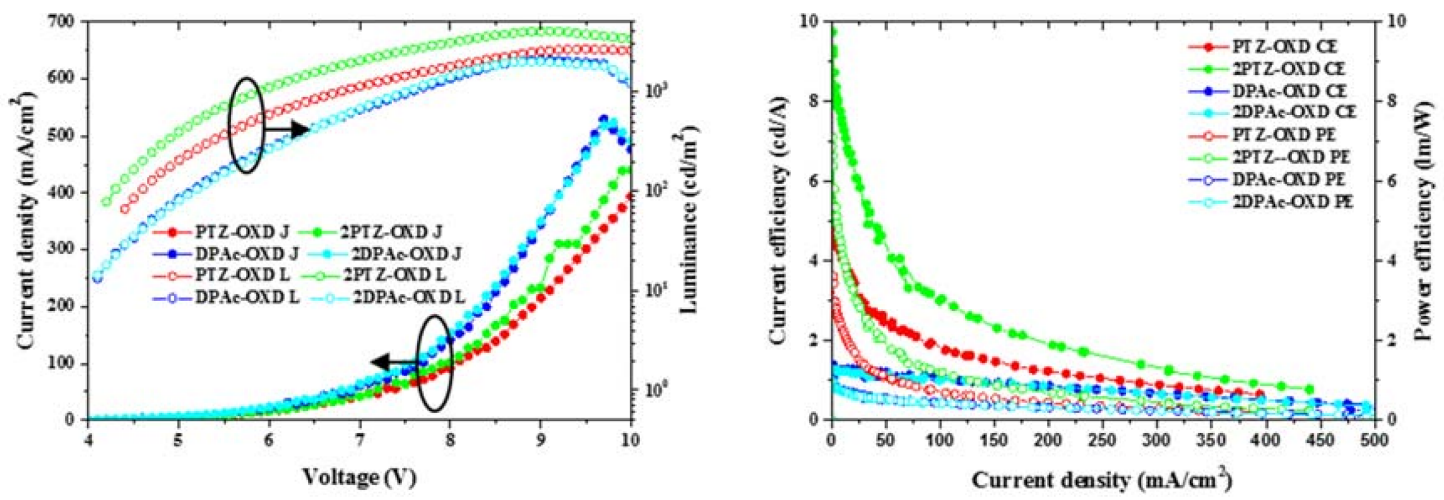

DEVICE 2
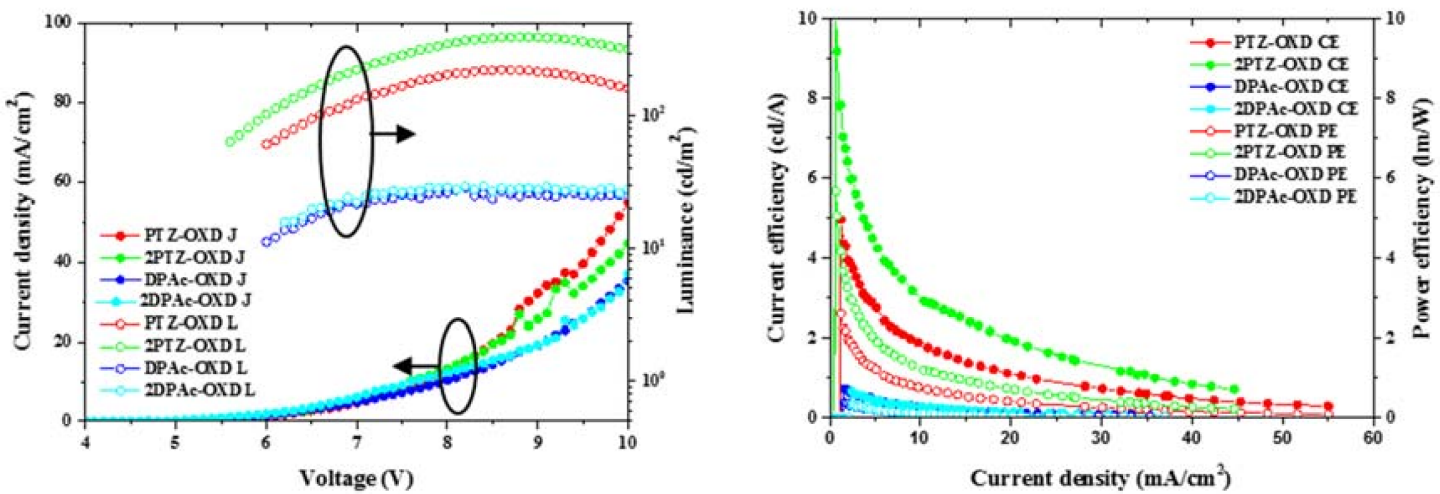

Figure 5. Current density-voltage-luminance $(J-V-L)$ and luminescence -current and power efficiency of the fabricated Devices 1 and 2.

Table 3. Device characteristics of fluorescent emitters PTZ-OXD, 2PTZ-OXD, DPAc-OXD and 2DPAc-OXD.

\begin{tabular}{ccccc}
\hline Device properties & PTZ-OXD & 2PTZ-OXD & DPAc-OXD & 2DPAc-OXD \\
\hline \multirow{2}{*}{ Turn on voltage (V) } & $4.4^{\mathrm{a}}$ & $4.2^{\mathrm{a}}$ & $4.1^{\mathrm{a}}$ & $4.1^{\mathrm{a}}$ \\
Driving voltage (V) & $6.0^{\mathrm{b}}$ & $5.6^{\mathrm{b}}$ & $6.0^{\mathrm{b}}$ & $6.2^{\mathrm{b}}$ \\
Current (mA) & $6.8^{\mathrm{a}}$ & $5.9^{\mathrm{a}}$ & $7.6^{\mathrm{a}}$ & $7.5^{\mathrm{a}}$ \\
& $0.05^{\mathrm{a}}$ & $0.03^{\mathrm{a}}$ & $0.04^{\mathrm{a}}$ & $0.05^{\mathrm{a}}$ \\
Current efficiency (cd/A) & $0.05^{\mathrm{b}}$ & $0.03^{\mathrm{b}}$ & $0.06^{\mathrm{b}}$ & $0.10^{\mathrm{b}}$ \\
& $5.03^{\mathrm{a}}$ & $9.20^{\mathrm{a}}$ & $1.30^{\mathrm{a}}$ & $1.28^{\mathrm{a}}$ \\
Power efficiency (Lm/W) & $4.97^{\mathrm{b}}$ & $10.10^{\mathrm{b}}$ & $0.72^{\mathrm{b}}$ & $0.66^{\mathrm{b}}$ \\
& $3.59^{\mathrm{a}}$ & $6.88^{\mathrm{a}}$ & $0.99^{\mathrm{a}}$ & $0.98^{\mathrm{a}}$ \\
EQE (\%) & $2.60^{\mathrm{b}}$ & $5.67^{\mathrm{b}}$ & $0.38^{\mathrm{b}}$ & $0.33^{\mathrm{b}}$ \\
Luminance (at $\left.1000 \mathrm{~cd} / \mathrm{m}^{2}\right)$ & $1.94^{\mathrm{a}}$ & $3.38^{\mathrm{a}}$ & $1.84^{\mathrm{a}}$ & $1.81^{\mathrm{a}}$ \\
CIE $(\mathrm{x}, \mathrm{y})$ & $2.26^{\mathrm{b}}$ & $3.99^{\mathrm{b}}$ & $1.08^{\mathrm{b}}$ & $0.88^{\mathrm{b}}$ \\
& 1008 & 1020 & 1029 & 992 \\
& $(0.38,0.50)^{\mathrm{a}}$ & $(0.40,0.53)^{\mathrm{a}}$ & $(0.16,0.12)^{\mathrm{a}}$ & $(0.17,0.14)^{\mathrm{a}}$ \\
& $(0.43,0.14)^{\mathrm{b}}$ & $(0.31,0.49)^{\mathrm{b}}$ & $(0.18,0.12)^{\mathrm{b}}$ & $(0.18,0.15)^{\mathrm{b}}$ \\
\hline
\end{tabular}

The electroluminescence study was carried out to find the emission wavelength and colour purity (Table 3). DPAc-OXD and 2DPAc-OXD showed similar emission peak at $450 \mathrm{~nm}$ along with CIE (x,y) colour coordinate of $(0.16,0.12)$ and $(0.17,0.14)$, respectively. The emission of PTZ-OXD was observed at $540 \mathrm{~nm}$, while 2PTZ-OXD showed emission at $550 \mathrm{~nm}$ with little bathochromic shift. We did not notice any other emissions other than our fluorescence dopants (Figure 6). 


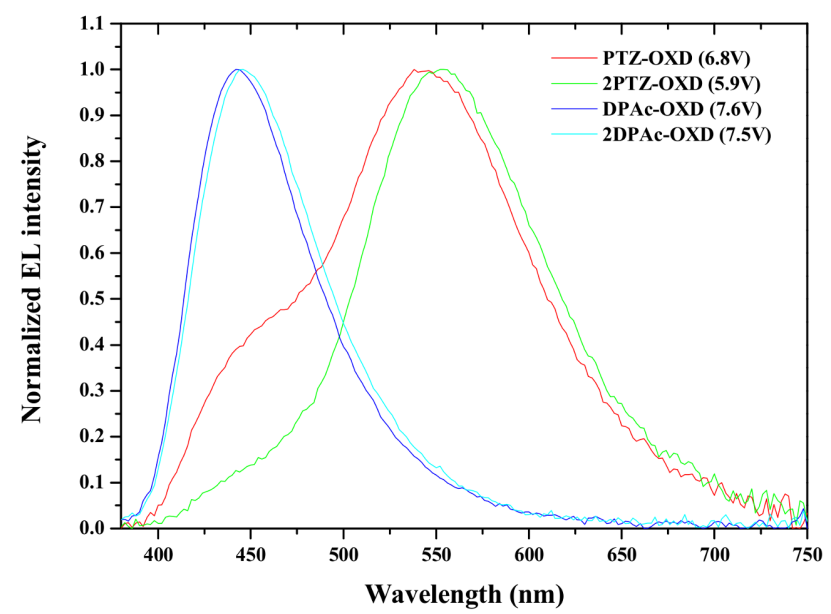

Figure 6. Normalized electroluminescent (EL) spectra of Device 1.

\section{Materials and Methods}

\subsection{General Procedures}

All reagents and solvent were purchased from commercial suppliers and were used without further purification otherwise stated. ${ }^{1} \mathrm{H}$ - and ${ }^{13} \mathrm{C}-\mathrm{NMR}$ (Nuclear Magnetic resonance) spectra were recorded by using a JNM-ECP FT-NMR spectrometer (JEOL, Peabody, MA, USA) and operating at $500 \mathrm{MHz}$. Absorbance spectra were recorded from a S-4100 UV-visible spectrophotometer (SINCO, Seoul, Korea). The Band gaps $\left(E_{\mathrm{g}}\right)$ were estimated from the onset of the absorbance spectra while photoluminescence (PL) spectra were measured by using a HR800 Spectro fluorimeter (Horiba Jobin Yvon, Paris, France). HOMO level was calculated by AC-2 using a Photoelectron spectrometer (RIKEN, Saitama, Japan). LUMO was calculated by subtraction of the band gap from the HOMO energy. Thermal gravimetric analysis was conducted on a DSC Q200 V24.9 Build 121 thermal analysis system (TA instruments, New castle, DE, USA) with the heating rate of $10^{\circ} \mathrm{C} / \mathrm{min}$. Mass analysis were carried out by using a Xevo TQ-S spectrometer (Waters, Milford, MA, USA). Current density-voltage-luminescence $(J-V-L)$ efficiencies were measured by an OLED I-V-L test system (Polarmix M6100, Suwon, Republic of Korea). The electroluminescence (EL) spectra analysis was carried out by using a spectroradiometer (Konica Minolta CS-2000, Japan). The molecular distributions were carried out by using a Gaussian 09 program (Wallingford, CT, USA) with DFT (density functional theory) and TD-SCF method B3LYP with a 6/31G basic set.

\subsection{Synthetic Procedures}

\subsubsection{4-Bromo- $N^{\prime}$-(4-bromobenzoyl)benzohydrazide (1)}

4-Bromobenzoyl chloride $(3.0 \mathrm{~g}, 13.67 \mathrm{mmol})$ was dissolved in $30 \mathrm{~mL}$ THF and stirred at $0{ }^{\circ} \mathrm{C}$ for $30 \mathrm{~min}$, then hydrazine monohydrate $(0.28 \mathrm{~mL}, 5.44 \mathrm{mmol})$ was added dropwise in to the mixture. After adding, the mixture was stirred for another $3 \mathrm{~h}$ at room temperature. The resulting precipitate was filtered, washed with saturated aqueous $\mathrm{NaHCO}_{3}$ solution $(3 \times 15 \mathrm{~mL})$ and water, respectively, and then dried to get white solid $(1.94 \mathrm{~g})$ with the yield of $84 \%$.

${ }^{1} \mathrm{H}$ NMR (500 MHz, DMSO-d 6 ) $\delta 10.65(\mathrm{~s}, 2 \mathrm{H}), 7.88-7.83(\mathrm{~m}, 4 \mathrm{H}), 7.75(\mathrm{dd}, J=6.42,4.53 \mathrm{~Hz}, 4 \mathrm{H})$.

\subsubsection{2,5-Bis(4-bromophenyl)-1,3,4-oxadiazole (2)}

4-Bromo- $N^{\prime}$-(4-bromobenzoyl)benzohydrazide( $\left.5 \mathrm{~g}, 12.56 \mathrm{mmol}\right)$ was placed in $250 \mathrm{~mL}$ two neck flask equipped with condenser. Then vacuumed it for $15 \mathrm{~min}$ and $30 \mathrm{~mL}$ of dry toluene and $30 \mathrm{~mL}$ 
$\mathrm{POCl}_{3}$ were injected. Under $\mathrm{N}_{2}$ atmosphere, the mixture was refluxed until the reaction was finished. After completion of the reaction, the solvent and excesses $\mathrm{POCl}_{3}$ were removed by a rotary evaporator under reduced pressure. Finally, the mixture was recrystallized with ethanol. The white crystals were filtered and dried $(4.75 \mathrm{~g}, 99.5 \%$ yield $)$.

${ }^{1} \mathrm{H}$ NMR $\left(500 \mathrm{MHz}, \mathrm{CDCl}_{3}\right) \delta 8.00(\mathrm{td}, J=8.00,1.72 \mathrm{~Hz}, 4 \mathrm{H}), 7.68(\mathrm{td}, J=7.99,1.70 \mathrm{~Hz}, 4 \mathrm{H}) ;{ }^{13} \mathrm{C} \mathrm{NMR}$ $\left(500 \mathrm{MHz} \mathrm{CDCl}_{3}\right) \delta 164.15,132.59,128.44,126.73,122.72$.

\subsubsection{2-(4-Bromophenyl)-5-phenyl-1,3,4-oxadiazole (4)}

The synthesis of 2-(4-bromophenyl)-1,3,4-oxadiazole is similar with OXD-2Br, and the only difference lies in the starting material 4-bromo- $N^{\prime}$-(4-bromobenzoyl)benzohydrazide to $N^{\prime}$-benzoyl-4-bromobenzohydrazide. After recrystallization, white crystals were filtered and dried in the oven.

Yield 93\%. ${ }^{1} \mathrm{H}$ NMR (500 MHz, $\left.\mathrm{CDCl}_{3}\right) \delta 8.15$ (dd, $\left.J=5.30,3.40 \mathrm{~Hz}, 2 \mathrm{H}\right), 8.04-7.99$ (m, 2H), 7.63-7.58 $(\mathrm{m}, 2 \mathrm{H}), 7.57-7.52(\mathrm{~m}, 1 \mathrm{H}), 7.52-7.46(\mathrm{~m}, 2 \mathrm{H}) ;{ }^{13} \mathrm{C} \mathrm{NMR}\left(500 \mathrm{MHz}, \mathrm{CDCl}_{3}\right) \delta 144.85,143.55,133.66$, $132.69,132.08,131.93,130.09,128.75,128.71,126.87$.

\subsubsection{2,5-Bis(4-(9,9-diphenyl-9,10-dihydroacridine)phenyl)-1,3,4-oxadiazole (2DPAc-OXD)}

2,5-Bis(4-bromophenyl)-1,3,4-oxadiazole (5 g, $13.16 \mathrm{mmol}), 9,9$-diphenyl-9,10-dihydroacridine $(9.64 \mathrm{~g}, 28.94 \mathrm{mmol})$, potassium carbonate $(12.04 \mathrm{~g}, 87.09 \mathrm{mmol})$ and palladium acetate $(0.18 \mathrm{~g}$, $0.79 \mathrm{mmol}$ ) were added in $500 \mathrm{~mL}$ two neck flask equipped with condenser and vacuumed it for $15 \mathrm{~min}$. Then $300 \mathrm{~mL}$ of dry toluene was injected. The mixture was continued with stirring and heating, after $10 \mathrm{~min} 7.3 \mathrm{~mL}$ tri-tert-butylphosphine (10\% in toluene) was injected. The above mixture was stirred and heated under reflux condition until the starting material disappear. After cooling to room temperature, the mixture was extracted with dichloromethane (DCM) and deionized water. Then the organic layer was dried over anhydrous $\mathrm{MgSO}_{4}$ and filtered. The organic layer was concentrated on a rotary evaporator under reduced pressure. Finally, the residue was purified by silica column chromatography to afford $8.76 \mathrm{~g}$ of (white solid) 2DPAc-OXD with the yield of $75.2 \%$.

${ }^{1} \mathrm{H}$ NMR $\left(500 \mathrm{MHz}, \mathrm{CDCl}_{3}\right) \delta 8.33-8.29(\mathrm{~m}, 4 \mathrm{H}), 6.46(\mathrm{~d}, J=8.24 \mathrm{~Hz}, 4 \mathrm{H}), 6.92(\mathrm{~d}, J=4.01 \mathrm{~Hz}, 8 \mathrm{H})$, $6.99(\mathrm{dd}, J=7.67,1.77 \mathrm{~Hz}, 8 \mathrm{H}), 7.08(\mathrm{td}, J=8.38,4.34,4.34 \mathrm{~Hz}, 4 \mathrm{H}), 7.29-7.21(\mathrm{~m}, 16 \mathrm{H}) ;{ }^{13} \mathrm{C} \mathrm{NMR}$ $\left(500 \mathrm{MHz}_{,} \mathrm{CDCl}_{3}\right) \delta 164.36,146.28,144.46,143.93,141.83,132.13,130.45,130.27,129.27,127.77,127.05$, 126.47, 120.78, 114.24, 108.01; MS (APCI): 886.09 for $\mathrm{C}_{64} \mathrm{H}_{44} \mathrm{~N}_{4} \mathrm{O}\left[\mathrm{M}+\mathrm{H}^{+}\right]$.

\subsubsection{2-(4-(9,9-Diphenyl-9,10-dihydroacridine)phenyl)-1,3,4-oxadiazole (DPAc-OXD)}

2-(4-bromophenyl)-5-phenyl-1,3,4-oxadiazole (3 g, $9.96 \mathrm{mmol}), 9,9$-diphenyl-9,10-dihydroacridine $(3.65 \mathrm{~g}, 1.96 \mathrm{mmol})$, potassium carbonate $(4.54 \mathrm{~g}, 32.87 \mathrm{mmol})$ and palladium acetate $(0.07 \mathrm{~g}, 0.299 \mathrm{mmol})$ were added in $250 \mathrm{~mL}$ two neck flask equipped with condenser and subjected it for $15 \mathrm{~min}$. Then $80 \mathrm{~mL}$ of dry toluene was injected, followed by $2.8 \mathrm{~mL}$ of tri-tert-butylphosphine $(10 \%$ in toluene) was added. After the completion of reaction, mixture was extracted with DCM and water. The organic layer was dried over anhydrous $\mathrm{MgSO}_{4}$ then filtered and evaporated under reduced pressure. The residue was purified by silica column chromatography to afford $4.7 \mathrm{~g}$ of DPAc-OXD as white solid with the yield of $85.4 \%$.

${ }^{1} \mathrm{H}$ NMR (500 MHz, $\left.\mathrm{CDCl}_{3}\right) \delta 8.33-8.29(\mathrm{~m}, 2 \mathrm{H}), 8.16(\mathrm{dd}, J=7.83,1.71 \mathrm{~Hz}, 2 \mathrm{H}), 7.61-7.55(\mathrm{~m}, 3 \mathrm{H})$, 7.31-7.24 (m, 8H), $7.08(\mathrm{ddd}, J=8.46,5.57,3.22 \mathrm{~Hz}, 2 \mathrm{H}), 7.00$ (dd, $J=7.89,1.70 \mathrm{~Hz}, 4 \mathrm{H}), 6.97-6.89$ $(\mathrm{m}, 4 \mathrm{H}), 6.46(\mathrm{~d}, J=8.05 \mathrm{~Hz}, 2 \mathrm{H}) ;{ }^{13} \mathrm{C} \mathrm{NMR}\left(500 \mathrm{MHz}, \mathrm{CDCl}_{3}\right) \delta 164.94,164.13,146.32,144.29,141.86$, 132.12, 131.99, 130.46, 130.27, 130.20, 129.25, 127.77, 127.09, 127.05, 126.47, 123.89, 123.70, 120.74, 114.22; MS (APCI): 553.75 for $\mathrm{C}_{39} \mathrm{H}_{27} \mathrm{~N}_{3} \mathrm{O}\left[\mathrm{M}+\mathrm{H}^{+}\right]$. 


\subsubsection{2,5-Bis(4-(10H-phenothiazin-10-yl)-1,3,4-oxadizole (2PTZ-OXD)}

2,5-Bis(4-bromophenyl)-1,3,4-oxadiazole (5 g, $13.15 \mathrm{mmol})$, phenothiazine ( $5.76 \mathrm{~g}, 28.93 \mathrm{mmol})$, potassium carbonate $(12.03 \mathrm{~g}, 87.05 \mathrm{mmol})$ and palladium acetate $(0.18 \mathrm{~g}, 0.79 \mathrm{mmol})$ were added in $250 \mathrm{~mL}$ two neck flask equipped with condenser and vacuumed it for $15 \mathrm{~min}$. Then $120 \mathrm{~mL}$ of dry toluene was injected while stirring. Then $7.3 \mathrm{~mL}$ of tri-tert-butylphosphine ( $10 \%$ in toluene) was injected in to the mixture. The above mixture was stirred and refluxed. After reaction completed, mixture was cooled to room temperature and extracted with dichloromethane (DCM) and deionized water. Then the organic layer was dried over $\mathrm{MgSO}_{4}$, followed by concentrated on a rotary evaporator under reduced pressure. Finally, the residue was purified by silica column chromatography to afford $7.88 \mathrm{~g}$ (yellow solid) 2PTZ-OXD with the yield of $97.2 \%$.

${ }^{1} \mathrm{H}$ NMR $\left(500 \mathrm{MHz}, \mathrm{CDCl}_{3}\right) \delta 8.17-8.13(\mathrm{~m}, 2 \mathrm{H}), 7.38-7.33(\mathrm{~m}, 2 \mathrm{H}), 7.28-7.22(\mathrm{~m}, 2 \mathrm{H}), 7.12(\mathrm{dt}, J=7.75$, $7.66,1.50 \mathrm{~Hz}, 2 \mathrm{H}), 7.05-7.02(\mathrm{~m}, 2 \mathrm{H}), 6.84(\mathrm{~d}, J=8.07 \mathrm{~Hz}, 2 \mathrm{H}),{ }^{13} \mathrm{C}$ NMR $\left(500 \mathrm{MHz}, \mathrm{CDCl}_{3}\right) \delta 160.15$, 146.50, 142.60, 128.88, 128.03, 127.47, 127.23, 124.65, 123.80, 121.54, 120.08; MS (APCI): 618.07 for $\mathrm{C}_{38} \mathrm{H}_{24} \mathrm{~N}_{4} \mathrm{OS}_{2}\left[\mathrm{M}+\mathrm{H}^{+}\right]$.

\subsubsection{2-(4-(10H-phenothiazin-10-yl)-1,3,4-oxadiazole(PTZ-OXD)}

2-(4-Bromophenyl)-5-phenyl-1,3,4-oxadiazole (3 g, $9.96 \mathrm{mmol})$, phenothiazine $(2.18 \mathrm{~g}, 10.96 \mathrm{mmol})$, potassium carbonate $(4.56 \mathrm{~g}, 32.98 \mathrm{mmol})$ and palladium acetate $(0.07 \mathrm{~g}, 0.299 \mathrm{mmol})$ were added in $250 \mathrm{~mL}$ two neck flask equipped with condenser and vacuumed it for $15 \mathrm{~min}$. Then $80 \mathrm{~mL}$ dry toluene was injected while stirring and $2.8 \mathrm{~mL}$ tri-tert-butylphosphine (10\% in toluene) was added. The mixture was stirred and refluxed until the reaction complete. After cooling to room temperature, the mixture was extracted with dichloromethane (DCM) and deionized water. The organic layer was dried over $\mathrm{MgSO}_{4}$, followed by concentrated on a rotary evaporator under reduced pressure. Finally, the residue was purified by silica column chromatography afford $4.18 \mathrm{~g}$ (light yellow solid) of PTZ-OXD with the yield of $98.5 \%$.

${ }^{1} \mathrm{H}$ NMR $\left(500 \mathrm{MHz}, \mathrm{CDCl}_{3}\right) \delta 8.33-8.29(\mathrm{~m}, 2 \mathrm{H}), 8.16(\mathrm{dd}, J=7.83,1.71 \mathrm{~Hz}, 2 \mathrm{H}), 7.61-7.55(\mathrm{~m}, 3 \mathrm{H})$, $7.31-7.24(\mathrm{~m}, 8 \mathrm{H}), 7.08(\mathrm{ddd}, J=8.46,5.57,3.22 \mathrm{~Hz}, 2 \mathrm{H}), 7.00(\mathrm{dd}, J=7.89,1.70 \mathrm{~Hz}, 4 \mathrm{H}), 6.97-6.89$ $(\mathrm{m}, 4 \mathrm{H}), 6.46(\mathrm{~d}, J=8.05 \mathrm{~Hz}, 2 \mathrm{H}) ;{ }^{13} \mathrm{C}$ NMR $\left(500 \mathrm{MHz}, \mathrm{CDCl}_{3}\right) \delta 164.94,164.13,146.32,141.86,132.12$, 131.99, 130.47, 130.27, 129.25, 127.78, 127.09, 127.05, 126.47, 123.70, 120.74, 114.22; MS(APCI): 419.80 for $\mathrm{C}_{26} \mathrm{H}_{17} \mathrm{~N}_{3} \mathrm{OS}\left[\mathrm{M}+\mathrm{H}^{+}\right]$.

\subsection{OLED Fabrication and Characterization}

The device substrate was made by using an ITO (indium tin oxide) with the thickness of $150 \mathrm{~nm}$. Then substrate was subjected to ultra-sonication with isopropyl alcohol and deionized water, followed by ultraviolet and ozone treatment. Further device fabrication continued as follow: 4,4'-bis( $N$-phenyl-1-naphthylamino)biphenyl (NPB) was used as hole transporting material (HTM), 1,3-bis(N-carbazolyl)benzene (mCP) was used for exciton blocking layer, bis[2-(diphenylphosphino)phenyl] ether oxide (DPEPO) and bis[2-(2-pyridinyl)phenolato]beryllium(II) (Bepp2) host materials doped with $6 \%$ fluorescence emitters, $2,2^{\prime}, 2^{\prime \prime}$-(1,3,5-benzinetriyl)-tris(1-phenyl-1- $H$-benzimidazole) (TPBI) was electron transporting material, 8-quinolinolato lithium (Liq) used for hole injecting material and aluminium was used for cathode with thickness of $100 \mathrm{~nm}$. All organic layers were deposited by thermal evaporating system under $5 \times 10^{-7}$ torr pressure (Sunicel plus, Seoul, Republic of Korea). The active area of the devices were $2 \mathrm{~mm} \times 2 \mathrm{~mm}$.

\section{Conclusions}

In summary, we have designed and synthesized four bipolar fluorescence emitters for OLEDs. All four materials were synthesized by using Buchwald-Hartwig amination with excellent yield 
over 75\%. Here, we used oxadiazole acceptor with acridine derivatives (DPAc-OXD, 2DPAc-OXD) and phenothiazine derivatives (PTZ-OXD, 2PTZ-OXD) to construct bipolar fluorescence materials. All four materials exhibited higher thermal stabilities which were expressed by thermal decomposition temperature at $5 \%$ weight reduction. In order to investigate the device efficiencies, we have fabricated two different devices with two different host materials Bepp2 (Device 1) and DPEPO (Device 2). The Device 1 performances were considerably higher than that of Device 2. The phenothiazine donor based 2PTZ-OXD emitter revealed the best current and power efficiencies of $10.10 \mathrm{~cd} / \mathrm{A}$ and $6.88 \mathrm{Lm} / \mathrm{W}$, respectively. The external quantum efficiency of 2PTZ-OXD was 3.99\%, while PTZ-OXD showed $2.26 \%$. When we compared the overall performances, 2PTZ-OXD exhibited excellent properties while acridine derivatives based 2DPAc-OXD and DPAc-OXD showed lower efficiencies due to its strong donating power. We found that balance charge donating and withdrawing nature were very important for bipolar fluorescence emitters in OLEDs to enhance the efficiencies.

Acknowledgments: This research was supported by basic science research program through the National Research Foundation of Korea (NRF) funded by the education (NRF-2016R1D1A3B01015531).

Author Contributions: K.Y.C. designed the project; Q.W. and H.Q.Z. performed experiment; R.B., I.J.B. and M.K. fabricated device and analyzed the data; R.B. and Q.W. wrote the manuscript.

Conflicts of Interest: The authors declare no conflict of interest.

\section{References}

1. Tang, C.W.; VanSlyke, S.A. Organic electroluminescent diodes. Appl. Phys. Lett. 1987, 51, 913-915. [CrossRef]

2. D'Andrade, B.W.; Forrest, S.R. White organic light-emitting devices for solid-state lighting. Adv. Mater. 2004, 16, 1585-1595. [CrossRef]

3. Reddy, S.S.; Sree, V.G.; Cho, W.; Jin, S.-H. Achieving pure deep-blue electroluminescence with CIE $y \leq 0.06$ via a rational design approach for highly efficient non-doped solution-processed organic light-emitting diodes. Chem. Asian J. 2016, 11, 3275-3282. [CrossRef] [PubMed]

4. Shirota, Y.; Kinoshita, M.; Noda, T.; Okumoto, K.; Ohara, T. A novel class of emitting amorphous molecular materials as bipolar radical formants: 2-\{4-[bis(4-methylphenyl)amino]phenyl\}-5-(dimesitylboryl)thiophene and 2-\{4-[bis(9,9-dimethylfluorenyl)amino]phenyl\}-5-(dimesitylboryl)thiophene. J. Am. Chem. Soc. 2000, 122, 11021-11022. [CrossRef]

5. Scott, J.C.; Karg, S.; Carter, S.A. Bipolar charge and current distributions in organic light-emitting diodes. J. Appl. Phys. 1997, 82, 1454-1460. [CrossRef]

6. Yamaguchi, Y.; Fujiyama, T.; Tanaka, H.; Yokoyama, M. Material design of organic thin films for bipolar charge transport. Chem. Mater. 1990, 2, 341-342. [CrossRef]

7. Woo, S.-J.; Kim, Y.; Kim, M.-J.; Baek, J.Y.; Kwon, S.K.; Kim, Y.H.; Kim, J.J. Strategies for the molecular design of donor-acceptor-type fluorescent emitters for efficient deep blue organic light emitting diodes. Chem. Mater. 2018, 30, 857-863. [CrossRef]

8. Reddy, S.S.; Sree, V.G.; Gunasekar, K.; Cho, W.; Gal, Y.S.; Song, M.; Kang, J.W.; Jin, S.H. Highly efficient bipolar deep-blue fluorescent emitters for solution-processed non-doped organic light-emitting diodes based on 9,9-dimethyl-9,10-dihydroacridine/phenanthroimadazole derivatives. Adv. Opt. Mater. 2016, 4, 1236-1246. [CrossRef]

9. Thirion, D.; Rault-Berthelot, J.; Vignau, L.; Poriel, C. Synthesis and properties of a blue bipolar indenofluorene emitter based on a D- $\pi$-A design. Org. Lett. 2011, 13, 4418-4421. [CrossRef] [PubMed]

10. Abdurahman, A.; Obolda, A.; Peng, Q.; Li, F. Efficient deep blue fluorescent oleds with ultra-low efficiency roll-off based on 4h-1,2,4-triazole cored D-A and D-A-D type emitters. Dyes Pigm. 2018, 153, 10-17. [CrossRef]

11. Liu, X.K.; Zheng, C.J.; Lo, M.F.; Xiao, J.; Chen, Z.; Liu, C.L.; Lee, C.S.; Fung, M.K.; Zhang, X.H. Novel blue fluorophor with high triplet energy level for high performance single-emitting-layer fluorescence and phosphorescence hybrid white organic light-emitting diodes. Chem. Mater. 2013, 25, 4454-4459. [CrossRef]

12. Lin, S.L.; Chan, L.H.; Lee, R.H.; Yen, M.Y.; Kuo, W.J.; Chen, C.T.; Jeng, R.J. Highly efficient carbazole- $\pi-$ dimesitylborane bipolar fluorophores for nondoped blue organic light-emitting diodes. Adv. Mater. 2008, 20, 3947-3952. [CrossRef] 
13. Fisher, A.L.; Linton, K.E.; Kamtekar, K.T.; Pearson, C.; Bryce, M.R.; Petty, M.C. Efficient deep-blue electroluminescence from an ambipolar fluorescent emitter in a single-active-layer device. Chem. Mater. 2011, 23, 1640-1642. [CrossRef]

14. Jeong, S.; Kim, M.K.; Kim, S.H.; Hong, J.I. Efficient deep-blue emitters based on triphenylamine-linked benzimidazole derivatives for nondoped fluorescent organic light-emitting diodes. Org. Electron. 2013, 14, 2497-2504. [CrossRef]

15. Ramaiah, D.; Thurakkal, S.; Sanju, K.S.; Soman, A.; Unni, N.; Joseph, J. Design and synthesis of solution processable green fluorescent D- $\pi$-A dyads for oled applications. New J. Chem. 2018, 42, 5456-5464.

16. Zhang, G.; Auer-Berger, M.; Gehrig, D.; Blom, P.; Baumgarten, M.; Schollmeyer, D.; List-Kratochvil, E.; Müllen, K. Blue light emitting polyphenylene dendrimers with bipolar charge transport moieties. Molecules 2016, 21, 1400. [CrossRef] [PubMed]

17. Uoyama, H.; Goushi, K.; Shizu, K.; Nomura, H.; Adachi, C. Highly efficient organic light-emitting diodes from delayed fluorescence. Nature 2012, 492, 234-238. [CrossRef] [PubMed]

18. Zhang, Q.; Kuwabara, H.; Potscavage, W.J.; Huang, S.; Hatae, Y.; Shibata, T.; Adachi, C. Anthraquinone-based intramolecular charge-transfer compounds: Computational molecular design, thermally activated delayed fluorescence, and highly efficient red electroluminescence. J. Am. Chem. Soc. 2014, 136, 18070-18081. [CrossRef] [PubMed]

19. Yook, K.S.; Lee, J.Y.; Yook, K.S. Bipolar host materials for organic light-emitting diodes. Chem. Rec. 2016, 16, 159-172. [CrossRef] [PubMed]

20. Zhu, Y.; Kulkarni, A.P.; Jenekhe, S.A. Phenoxazine-based emissive donor-acceptor materials for efficient organic light-emitting diodes. Chem. Mater. 2005, 17, 5225-5227. [CrossRef]

21. Goes, M.; Verhoeven, J.W.; Hofstraat, H.; Brunner, K. Oled and pled devices employing electrogenerated, intramolecular charge-transfer fluorescence. ChemPhysChem 2003, 4, 349-358. [CrossRef] [PubMed]

22. Kulkarni, A.P.; Kong, X.; Jenekhe, S.A. High-performance organic light-emitting diodes based on intramolecular charge-transfer emission from donor-acceptor molecules: Significance of electron-donor strength and molecular geometry. Adv. Funct. Mater. 2006, 16, 1057-1066. [CrossRef]

23. Zhong, H.; Lai, H.; Fang, Q. New conjugated triazine based molecular materials for application in optoelectronic devices: Design, synthesis, and properties. J. Phys. Chem. C 2011, 115, 2423-2427. [CrossRef]

24. Linton, K.E.; Fisher, A.L.; Pearson, C.; Fox, M.A.; Palsson, L.O.; Bryce, M.R.; Petty, M.C. Colour tuning of blue electroluminescence using bipolar carbazole-oxadiazole molecules in single-active-layer organic light emitting devices (oleds). J. Mater. Chem. 2012, 22, 11816-11825. [CrossRef]

25. Duan, L.; Qiao, J.; Sun, Y.; Qiu, Y. Strategies to design bipolar small molecules for oleds: Donor-acceptor structure and non-donor-acceptor structure. Adv. Mater. 2011, 23, 1137-1144. [CrossRef] [PubMed]

26. Li, Y.; Li, A.Y.; Li, B.-X.; Huang, J.; Zhao, L.; Wang, B.-Z.; Li, J.-W.; Zhu, X.-H.; Peng, J.; Cao, Y.; et al. Asymmetrically 4,7-disubstituted benzothiadiazoles as efficient non-doped solution-processable green fluorescent emitters. Org. Lett. 2009, 11, 5318-5321. [CrossRef] [PubMed]

27. Ye, J.; Chen, Z.; Fung, M.K.; Zheng, C.; Ou, X.; Zhang, X.; Yuan, Y.; Lee, C.S. Carbazole/sulfone hybrid D- $\pi$-A-structured bipolar fluorophores for high-efficiency blue-violet electroluminescence. Chem. Mater. 2013, 25, 2630-2637. [CrossRef]

28. Zhang, M.; Xue, S.; Dong, W.; Wang, Q.; Fei, T.; Gu, C.; Ma, Y. Highly-efficient solution-processed oleds based on new bipolar emitters. Chem Comm 2010, 46, 3923-3925. [CrossRef] [PubMed]

29. Antoniadis, H.; Inbasekaran, M.; Woo, E.P. Blue-green organic light-emitting diodes based on fluorene-oxadiazole compounds. Appl. Phys. Lett. 1998, 73, 3055-3057. [CrossRef]

30. Lian, M.; Yu, Y.; Zhao, J.; Huang, Z.; Yang, X.; Zhou, G.; Wu, Z.; Wang, D. Novel phosphorescent polymers containing both ambipolar segments and functionalized Ir ${ }^{\mathrm{III}}$ phosphorescent moieties: Synthesis, photophysical, redox, and electrophosphorescence investigation. J. Mater. Chem. C 2014, 2, 9523-9535. [CrossRef]

31. Wróblowska, M.; Kudelko, A.; Kuźnik, N.; Łaba, K.; Łapkowski, M. Synthesis of extended 1,3,4-oxadiazole and 1,3,4-thiadiazole derivatives in the Suzuki cross-coupling reactions. J. Heterocycl. Chem. 2017, 54, 1550-1557. [CrossRef]

32. Kwon, W.; Ahn, B.; Kim, D.M.; Ko, Y.-G.; Hahm, S.G.; Kim, Y.; Kim, H.; Ree, M. Morphology-dependent electrical memory characteristics of a well-defined brush polymer bearing oxadiazole-based mesogens. J. Phys. Chem. C 2011, 115, 19355-19363. [CrossRef] 
33. Mi, B.; Gao, Z.; Liao, Z.; Huang, W.; Chen, C.H. Molecular hosts for triplet emitters in organic light-emitting diodes and the corresponding working principle. Sci. China Chem. 2010, 53, 1679-1694. [CrossRef]

34. Zhao, F.; Zhang, Z.; Liu, Y.; Dai, Y.; Chen, J.; Ma, D. A hybrid white organic light-emitting diode with stable color and reduced efficiency roll-off by using a bipolar charge carrier switch. Org. Electron. 2012, 13, 1049-1055. [CrossRef]

35. Zhang, J.; Ding, D.; Wei, Y.; Xu, H. Extremely condensing triplet states of DPEPO-type hosts through constitutional isomerization for high-efficiency deep-blue thermally activated delayed fluorescence diodes. Chem. Sci. 2016, 7, 2870-2882. [CrossRef]

36. Braveenth, R.; Bae, I.-J.; Han, J.-H.; Qiong, W.; Seon, G.; Raagulan, K.; Yang, K.; Park, Y.H.; Kim, M.; Chai, K.Y. Utilizing a Spiro Core with Acridine- and Phenothiazine-Based New Hole Transporting Materials for Highly Efficient Green Phosphorescent Organic Light-Emitting Diodes. Molecules 2018, 23, 713. [CrossRef] [PubMed]

37. Song, D.; Zhao, S.; Luo, Y.; Aziz, H. Causes of efficiency roll-off in phosphorescent organic light emitting devices: Triplet-triplet annihilation versus triplet-polaron quenching. Appl. Phys. Lett. 2010, 97, 268. [CrossRef]

38. Zheng, T.; Choy, W.C.; Ho, C.L.; Wong, W.Y. Improving efficiency roll-off in organic light emitting devices with a fluorescence-interlayer-phosphorescence emission architecture. Appl. Phys. Lett. 2009, 95, 264. [CrossRef]

Sample Availability: Samples of the compounds are not available from the authors. 